\title{
The Reliability Analysis of Bridge Structure Under Multipoint Excitation
}

\author{
Fanghua Liu and Hezhi Li
}

Nanchang Key Laboratory of Material and Structure Detection, Jiangxi University of Technology

Keywords: Seismic Response; Structure Reliability; Genetic Algorithm; Multipoint Excitation; Response Spectrum

\begin{abstract}
With the large-scale development of China's economy and transportation in recent years, more and more long-span bridges are built and put into use. The scale of bridges becomes more and more big. The tower large span cable-stayed bridges are commonly seen. As one of the lifeline engineering, the seismic design and the reliability under seismic situation are particularly important. This paper first introduces the research situation and the theory of the reliability as well as some calculation methods used in the application of bridge reliability calculation. Based on the structure bearing capacity, the normal use of structure and structure system reliability, this paper explains the reliability process both at home and abroad. At the same time, this paper also discusses the response spectrum analysis method of the stochastic response of large span structure under the incentive function of multi-support seismic. In addition, this paper deduces multipoint excitation response spectrum combination formula according to the literature, and calculates the mean and standard deviation of the peak response of bridge structure. Finally, taking the cable-stayed bridge as an example, this paper establishes the analysis model, carries out the modal analysis, static analysis and dynamic analysis of bridges, and conducts the calculation analysis of bridge reliability under multipoint excitation based on the above methods.
\end{abstract}

\section{Introduction}

Earthquake is a natural phenomenon that happens many times every year all over the world. The vast majority of earthquakes cause no harm to people's safety or engineering construction. The earthquakes with harm happen more than a dozen times in a year on average all over the world. From the experience both at home and abroad, the earthquake damage to bridges is serious. In the lifeline engineering, since its structure can afford long-span stress, its appearance is aesthetically pleasing, its cost is moderate and its construction is convenient, the cable-stayed bridge has now become one of the most widely used form of long-span bridges of the world. In recent decades, because of the development of the design theory, construction methods and new materials research of the cable-stayed bridge, the worldwide cable-stayed bridge construction also processes a rapid growth in number. Once the long-span cable-stayed bridge usually serving as bridge transportation hub suffers from severe damage in an earthquake, not only huge direct losses will be caused, as a part of the lifeline engineering, its damage will cause problems to the disaster relief which makes the secondary disasters be more serious. Therefore, the correct and effective seismic reliability analysis of cable-stayed bridges has important economic and practical significances to ensure the anti-seismic safety.

Since the 1920s, the research on the basic theory of structural reliability has been carried out and gradually extended to all aspects of the structural analysis and design; until the 1970s, the application 
of the reliability method in structural design specification has become an important part of the study of reliability. From the 1950s, the institutions of higher learning and scientific research units of China carried out the research and discussion of the limit state method, which is to study the probability distribution of load and material strength and to determine the overload coefficient and materials (steel and concrete) intensity uniformity coefficient.

At home and abroad, the study of the reliability used by bridge structure system is not mature. Since there are a lot of structural components involved in the research of the bearing capacity, the normal use function and life prediction of the bridge structure, such as the upper bearing component, the upper general components, the pier body and foundation, the bearing and bridge deck, etc. What's more, there are variety of damage form s(or damages) involved in each component, such as bending, shearing and durability's not meeting requirements causing by cracking, etc. At the same time, the influence of these factors cannot be quantified precisely in the analysis and calculation of reliability. As a result, it is difficult to carry out the research of the bearing capacity and normal use of reliability of bridges. Although it is theoretically feasible, the actual operation is difficult, especially the research of the time-variant reliability of bridges which has more unknown parameters. There is no effective method available until now.

This paper creatively develops the theory of stochastic structure analysis, puts forward the rule and algorithm of random structure modeling and originally establishes the seismic reliability analysis theory of industrial production system. It also makes contributions to the improvement of reliability calculation and the reliability analysis of bridge structural system under seismic action.

\section{Introduction to Genetic Algorithm}

In recent years, with the widespread application of electronic computers in the field of engineering, Genetic Algorithm is introduced to the engineering. Genetic Algorithm is a kind of adaptive global optimization probability search algorithm, which simulates genesis and evolution of biology in natural environment. It makes a perfect combination of biological evolution theory and optimization technology to computer technology, which overcomes the disadvantage of the traditional optimization algorithm, and is easy to fall into local minimum value. It has created a new optimization method. With its excellent calculation performance and its obvious application effect, it is widely applied and focused on in various industries and multiple disciplines of national economy.

With its remarkable characteristics, such as simplicity and generality, being suitable for parallel processing and high efficiency, genetic algorithm has been widely applied in different fields, and achieved good results. As a new optimization technique, genetic algorithm has been widely concerned. For the features of genetic algorithm, the advantages of using genetic algorithm to solve problems are as follows:

Genetic algorithm uses the natural evolution mechanism to show the complex phenomenon and takes the coding of decision variables as the operation objects. The coding processing method makes the optimization process draw lessons from the conceptions of chromosomes and genes in biology, and imitates the heredity and evolution mechanism of creatures in nature.

Genetic algorithm only uses the value of fitness function to evaluate genetic individual on the basis of processed genetic operation, which is not only bound by continuous differentiation, and the domain can be set at will and search information with the objective function values. This feature is extremely convenient to process optimization problems of functions for the ones whose objective function is unable or difficult to differentiate. 
The group search feature of genetic algorithm makes it have better global searching ability and also makes it is easy to parallelize. Moreover, the search of genetic algorithm is not easy to fall into local optimum. Genetic algorithm has scalability and it is easy to be mixed with other techniques, which makes it be widely used in engineering field.

\section{Adoption of Genetic Algorithm in the Reliability Analysis of Bridge Structure}

In the genetic algorithm, the coding mode determines the precision, difficulty and the interpretation problems. The commonly used coding schemes are binary coding, gray coding, dynamic parameter coding, multi-value coding, real value coding and interval coding, etc. Among these codes, each has advantages and disadvantages. There is no a theory to jud ge the various coding methods and to guide their design. Binary coding is widely used due to the relatively easy imple ment. But the disadvantage is discontinuous, because the binary codes of two neighboring points in Euclidean space are not nearby in Hamming distance. Gray coding make this problem be solved.

Choice is the main mechanism of genetic algorithm and is also the most important factor influencing the performances of genetic algorithm. Selection operator is to choose an individual in a group. It is a random map $T_{S}: S^{N} \rightarrow S$. The usual method is, according to the probability rules, $T_{S}: S^{N} \rightarrow S P\left\{T_{S}^{a}(\bar{X})\right\}=\left\{X_{i}\right\}=\frac{f^{a}\left(x_{i}\right)}{\sum_{k=1}^{N} f^{a}\left(x_{k}\right)}$

$$
\text { choose. Here, } \bar{X}=\left(X_{1}, X_{2} \cdots X_{n}\right), f\left(X_{i}\right) \text { says the }
$$

fitness of the individual in group, $0<a<\infty$. When $a=1$, it is called selection operator which is short for

$T_{s}$. In selection operator, the individual with high adaptive value is easy to be selected. Selection operator is to choose in a fixed group, and therefore the better individuals outside the group are impossible be chosen. Commonly used selection operators are rank selections, rule selections and the transform of the scale of fitness function, etc.

If $X_{i}(t)$ shows the $\mathrm{i}$ individual of $\mathrm{t}$ generation group, the iterative process of the standard genetic algorithm can be described as:

Step 1: $\mathrm{t}=0$, initial groups are randomly generated $X(0)=\left\{X_{1}(0), X_{2}(0) \cdots X_{n}(0)\right\}$;

Step2: Independently choose $\mathrm{N}$ pairs of matrix from the current group;

Step3: Independently carry out hybridization to the chosen $\mathrm{N}$ pairs of matrix and produce $\mathrm{N}$ intermediate individuals;

Step 4: Independently carry out variation to $\mathrm{N}$ intermediate individuals and produce $(\mathrm{t}+1)$ generation group.

$$
X(t+1)=\left\{X_{1}(t+1), X_{2}(t+1) \cdots X_{n}(t+1)\right\}
$$

Step 5: Stop or set $\mathrm{t}=\mathrm{t}+1$, and return to Step 2.

The commonly used termination conditions of genetic algorithm are: (1) the specific genetic algebra t terminates after a certain generations. (2) Taking the fitness of chromosomes as conditions, when the fitness of chromosome reaches to a specified value, then terminates. (3) When all the 
chromosomes in a group reach to a certain similar degree (most chromosomes have similar bite at many positions), then terminates.

It can be seen from the standard genetic algorithm, as a kind of adaptive random search method, the information of genetic algorithm is provided by the current group, which is not determined by the single direction or structure. At the same time, it takes more individuals as possible solutions and considers searching the samples of the whole scope in the space, which leads to a greater possibility of convergence to the optimal solution whole scope. Due to these features, the genetic algorithm can be successfully used in many optimization problems which are different, complex and difficult to solve, including numerical optimization problems.

\section{The Basic Theory of Structural Reliability}

As one of the lifeline projects, bridge engineering has different characteristics compared with other artificial products, such as high construction costs (small scale project will cost ten million Yuan while large scale project will cost hundreds of billions Yuan) and long cycle (short cycle will be several years while long cycle will be hundreds of years). As infrastructure, bridge engineering is not only related to the national economy and people's livelihood, it will also affect the modernization of a country. Therefore, to ensure the structure can afford all kinds of function, meet the design requirements and keep its performance ability without too much maintenance in use cycle is crucial, which is to guarantee the safety, applicability and durability of the structure. These three aspects constitute the basic contents of bridge structure reliability.

The probability of structure or structural elements complete reservation function $(Z \geq 0)$ is called the reliable probability, which is also called reliability $\left(p_{s}\right)$; while the probability that cannot complete the function $(\mathrm{Z}<0)$ is known as the failure probability $\left(p_{f}\right)$. Set performance function only has relation with two basic variables, which are action effect $S$ (a variety of internal force and deformation, etc. caused by the action of the structure) and structure resistance $\mathrm{R}$ (the resistance of structural damage or deformation, such as extreme stress, ultimate strength and stiffness and sliding resistance, anti overturning moment, etc.), the structure function is:

$$
Z=g(R, S)=R-S
$$

And the corresponding limit state equation is:

$$
\mathrm{Z}=\mathrm{R}-\mathrm{S}=0
$$

As shown in Fig. 1, the limit state equation $\mathrm{R}-\mathrm{S}=0$ is a straight line, which axis 45 degrees with Axle $R$ and Axle $S$ on plane $(R, S)$. Because of its simple form, it is very convenient to determine the required integral domain. As functions of the basic variables, their distribution or joint distribution of $\mathrm{R}$ and $\mathrm{S}$ can be determined according to the function of a random variable in theory, however, it is difficult in practice. Because the distribution of the sum and product of random variables can be determined by normal distribution or logarithmic normal distribution according to central limit theorem. However, R and S are functions which are generally in the form of the sum or product of basic variables. As a result, on engineering problems, sometimes a kind of the above can be approximately chosen as a distribution pattern according to the actual situation. If the degree of approximation is not satisfied, another distribution which is more reasonable can be draft through 
Monte Carlo simulation method of function further according to the known distribution of basic variables.

Now according to the probability distribution of $\mathrm{R}$ and $\mathrm{S}$, respectively explain the calculation method of reliability or failure probability. Both $\mathrm{R}$ and $\mathrm{S}$ obey normal distribution and the mean values and the standard deviations are $\mu_{R}, \mu_{S}$ and $\sigma_{R}, \sigma_{S}$. The function $\mathrm{Z}$ is the new function composed by the two random variables $\mathrm{R}$ and $\mathrm{S}$. According to the theory of probability, $\mathrm{Z}$ also obeys the normal distribution and the mean value and the standard deviation are $\mu_{Z}=\mu_{R}-\mu_{S}$ and $\sigma_{Z}=\sqrt{\sigma_{R}^{2}+\sigma_{S}^{2}}$. The probability density function is:
$f_{Z}(Z)=\frac{1}{\sqrt{2 \pi \sigma}} \exp \left[-\frac{1}{2}\left(\frac{Z-\mu_{Z}}{\sigma_{Z}}\right)^{2}\right]-\infty<Z<+\infty$

\section{The response spectrum analysis of the reliability of seismic structure in seismic reaction}

For the dynamic analysis of elastic structure under consistent seismic incentive action, the classical response spectrum combination theory includes SRSS method, completely combination formula CQC, DSC, etc. Due to their fewer amounts of calculation, calculation accuracy and other advantages, they have been accepted and used by the design engineer. But, strictly speaking, these formulas can only give the maximum average of the structure seis mic response but not the maximum response variance. Therefore, it is difficult to implement the correct structure seismic reliability analysis. In 1981, according to random vibration theory, Kiureghian firstly deduced the variance formula of based on the structure random response of response spectrum. The basic formula provided opportunity for response spectrum theory to be reasonable used in structure seismic reliability analysis. According to the theory, Professors Li Jie, Li Jianhua and Doctor Ding Guangying further developed the practical method for the seismic reliability analysis of structure, artificially simulated seismic wave and finally obtained the formula of the superior performance

During the period of severe vibration, the earthquake acceleration records usually contain a period when the strength is approximately unchanged. As a result, a limited duration of white noise can be considered to use to simulate the process of this paragraph. Zero mean stationary Gaussian white noise was the earliest random model adopted and the density function of its power can be shown as:

$$
S_{\omega}=S_{0}(-\infty \leq \omega \leq+\infty)
$$

$S_{0}$ is a constant. It can be seen that the frequency distribution of white noise model is uniform in the scope of plus or minus infinity and the variance is unlimited, which is not consistent with the actual earthquake. But because it assumes that the seismic power spectrum is a certain value, it is easy to handle in mathematics and it is more likely to get the analytical solution. As a result, it is still used.

Due to the frequency of the seismic process always distributes over a limited range, some scholars improved the while noise model and put forward the limited bandwidth white noise model. The power spectral density function is: 


$$
S_{\omega}=S_{0}\left(-\omega_{0} \leq \omega \leq+\omega_{0}\right)
$$

It can be seen that the new model avoids the unreasonable phenomenon that the variance of the while noise model is unlimited.

\section{Conclusion}

The main contribution of this paper is to apply genetic algorithm and multipoint excitation response spectrum method in the structural reliability analysis of bridges. Genetic algorithm improved the traditional method of simple and two ranks quadrature. Then this paper deduces the multipoint excitation combination function that benefits the simplified coefficients, which has important practical significances to reliability analysis of bridges and the research of the reliability analysis of bridges.

According to the related theory, using the geo metric meaning of $\beta$ which is the reliability index in the method of simple and two ranks quadrature of reliability establish optimization model and introduce genetic algorithm for the reliability analysis of bridges. The calculation results show that, under the situation of selecting appropriate operators and boundary conditions, the individual can achieve better convergence effect and accuracy requirement, and is not influenced by functional form.

According to the related literature, deduce the theoretical basis of "multipoint excitation combination formula" method and use some simplified coefficients to participate in calculation. Proved by examples, the method recommended by this paper can better simulate the multipoint excitation of bridge structure, and simplify a coefficient which greatly improves calculation efficiency and precision.

Through the study of the reliability analysis of cable-stayed bridges, under different wave velocity, this paper calculates the maximum response and the standard deviation of the key position of the main girder and cable tower. Under the condition of the different distribution forms, according to the force majeure and response function, this paper calculates the structure reliability in bending and shearing modes.

\section{Acknowledgment}

This work was supported by Project on professional and characteristic construction of Jiangxi province 2010 (Civil Engineering) and Project on the planning and construction of disciplines in Jiangxi University of Technology (Structure Engineering)

\section{References}

[1] DING Y, LIN W, LI Z. Non-stationary Random Seismic Analysis of Long-span Spatial Structures under Multi-support and Multi-dimensional Earthquake Excitations [J][J]. Engineering Mechanics, 2007, 3: 016.

[2] Housner G W, Bergman L A, Caughey T K, et al. Structural Control: Past, Present, and Future [J]. Journal of Engineering Mechanics, 1997, 123(9): 897-971.

[3] Der Kiureghian A, Lin H Z, Hwang S J. Second-order reliability approximations [J]. Journal of 
Engineering Mechanics, 1987, 113(8): 1208-1225.

[4] Ni Y Q, Xia Y, Liao W Y, et al. Technology Innovation in Developing the Structural Health Monitoring System for Guangzhou New TV Tower [J]. Structural Control and Health Monitoring, 2009, 16(1): 73-98.

[5] Zi X. An Efficient Algorithm for Multi-dimensional and Multi-support Random Seismic Analysis of Spatial Reticulated Structures [J][J]. World Information on Earthquake Engineering, 2004, 3.

[6] LI Z, HUANG J, DING Y, et al. Seismic Responses of Long-span Cable-stayed Bridges under Different Patterns of Earthquake excitations [J]. China Journal of Highway and Transport, 2005, 18(3): 48-53.

[7] Xianming L. A Simplified Method for the Simulation of the Time Histories of Spatial Correlative Multi-Point Ground Motion [J][J]. Earthquake Resistant Engineering, 2003, 1: 008.

[8] Shunzai L A C H H. THE RELIABILITY ANALYSIS OF EARTHQUAKE-RESISTANT ARCH DAM SUBJECTED TO RANDOM MULTI-SUPPORT INPUT [J][J]. Earthquake Engineering and Engineering Vibration, 1996, 1: 004.

[10] HUANG X, HU D, ZHANG H. Effects of Traveling Wave to Seismic Response of Long Span Rigid-framed Bridge [J][J]. Journal of Chang'an University (Natural Science Edition), 2008, 1: 015 .

[11] Jiahao L, Wanxie Z. Some Notes on FEM and Structural Random Response Analysis [J][J]. Chinese Journal of Computational Mechanics, 1998, 2. 